\title{
Inverted Duplication and Deletion of 10q25q26 in a Patient without Any Obvious Skeletal Anomalies
}

\author{
B. Xiao X. Ji Y.Xing W.-T. Jiang J.-M.Zhang J.Tao
}

Xinhua Hospital, Shanghai Jiaotong University School of Medicine and Department of Genetics, Shanghai Institute for Pediatric Research, Shanghai, China

\section{Key Words}

Array-CGH • Chromosome 10 - Developmental delay ·

Duplication $\cdot$ Skeletal anomalies

\begin{abstract}
We report on a girl with inverted duplication and deletion of $10 q 25 q 26$ revealed by array-CGH and FISH analysis. ArrayCGH analysis demonstrated a $\sim 13.1-\mathrm{Mb}$ duplication encompassing 10q25.3q26.2 and a $\sim 5-\mathrm{Mb}$ deletion at 10q26.2q26.3. No single-copy region was detected between the deleted and duplicated segments. FISH analysis found the arrangement duplicated in an inverted position. FISH analysis using the same probes did not show any abnormality in both parents, which indicates a de novo occurrence. The frequently reported features of distal 10q duplication include developmental delay, blepharophimosis, hypotonia, skeletal anomalies and some facial dysmorphisms. The girl presented with many features of distal 10q duplication with the exception of skeletal anomalies. To our knowledge, this is the fourth patient reported in the literature with inv dup del 10q. 10q duplication seems to account for most of the phenotypes for our patient. Although no obvious skeletal feature was found in our patient at present, follow-up assessment of skeletal development should be planned with the increase of age.
\end{abstract}

Copyright $\odot 2012$ S. Karger AG, Basel
Inverted duplication and terminal deletion were first described by Weleber et al. [1976]. Usually, it is difficult to interpret well this rearrangement by conventional karyotype analysis. The wide use of molecular methods has allowed us to better elucidate and understand these complex rearrangements, and a number of chromosomes with this rearrangement have been identified.

Inverted duplication and concomitant deletion of $10 \mathrm{q}$ (inv dup del 10q) is rare. Three patients with inv dup del 10q have been reported recently [Carter et al., 2010; Kibe et al., 2011; Sarri et al., 2011]. In this report, we describe the clinical and molecular data of a patient with inv dup del $10 \mathrm{q}$ revealed by array-CGH and FISH, and compare them with other patients with molecularly defined distal 10q rearrangements.

\section{Patient and Methods}

\section{Clinical Report}

Our patient was the first child born to healthy non-consanguineous Chinese Han parents following a gestation of 40 weeks and 5 days (fig. 1a). Both parents were 30 years old at the delivery. Family history was unremarkable. There was no history of exposure to any teratogenic agent, illness and drug. The birth weight was $2,700 \mathrm{~g}$ (3rd-15th centile), the length was $50 \mathrm{~cm}$ (50th-85th centile). She could not sit unaided when she was admitted to our

\section{KARGER}

Fax +41613061234

E-Mail karger@karger.ch

www.karger.com (c) 2012 S. Karger AG, Basel

$1661-8769 / 12 / 0034-0185 \$ 38.00 / 0$

Accessible online at:

www.karger.com/msy
Jiong Tao

Shanghai Institute for Pediatric Research

Xinhua Hospital Affiliated to Shanghai Jiaotong University School of Medicine

1665 Kongjiang Road, Shanghai 200092 (China)

E-Mail tao1970@yahoo.cn 
hospital at 11 months. On examination at this age, her length was $68 \mathrm{~cm}$ ( $3 \mathrm{rd}-15$ th centile), weight $4,000 \mathrm{~g}$ ( $<3$ rd centile), and head circumference was $43.8 \mathrm{~cm}$ (15th-50th centile). The clinical features include hypertelorism, bilateral blepharophimosis, epicanthal folds, depressed flat nasal bridge, small nose, anteverted nostrils, long philtrum, convergent strabismus and hypotonia. A moderate laxity of both shoulder joints was noted. Heart auscultation did not reveal any significant murmurs. Brain CT scan was normal. Hip joint X-ray was unremarkable. On the latest examination at the age of 32 months (fig. 1b), length was $90 \mathrm{~cm}$ (15th50 th centile), weight $12.5 \mathrm{~kg}$ (15th-50th centile), and head circumference was $49 \mathrm{~cm}$ (50th-85th centile). She could walk with aid and speak a few words.

\section{Cytogenetic Investigations}

Routine karyotype analysis of the patient and her parents was performed on GTG-banded metaphases from cultures of PHAstimulated peripheral blood lymphocytes according to standard procedures.

\section{Array-CGH}

The proband was analyzed with the Agilent $4 \times 44 \mathrm{k}$ CGH array (Agilent Technologies, Palo Alto, Calif., USA). Genomic DNA was prepared from peripheral blood. The array experiment was performed using standardized protocols provided by the manufacturer. Genomic workbench standard edition 5.0.14 (Agilent Technologies) was used to plot the $\log _{2}$ ratio of signal intensity of each probe across the whole genome. The normalized ratios were analyzed for loss and gain of regions by an Aberration Detection Method 2 algorithm and the threshold was set to 6.0. The chromosome positions were shown according to the NCBI build 36.1 (hg18).

\section{FISH}

FISH analysis was performed on metaphases prepared from peripheral blood lymphocytes obtained by standard protocols. In order to identify the structural rearrangements, human BAC clones selected from the UCSC genome browser (http://www.genome.ucsc.edu) were used to identify the structural rearrangements. These clones were provided by the BAC/PAC Resources Center (Oakland, Calif., USA).
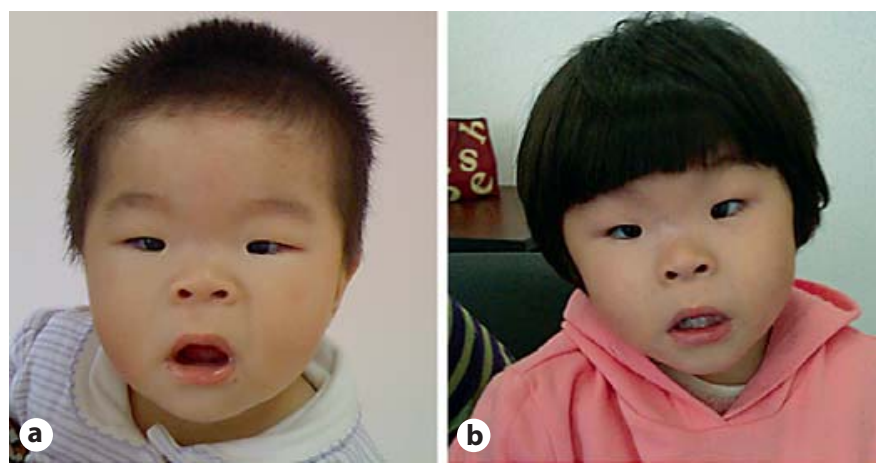

Fig. 1. Photograph of the patient. a Frontal view at 11 months of age. b Frontal view at 32 months of age. Note hypertelorism, bilateral blepharophimosis, epicanthal folds, depressed flat nasal bridge, small nose, anteverted nostrils, long philtrum, convergent strabismus.

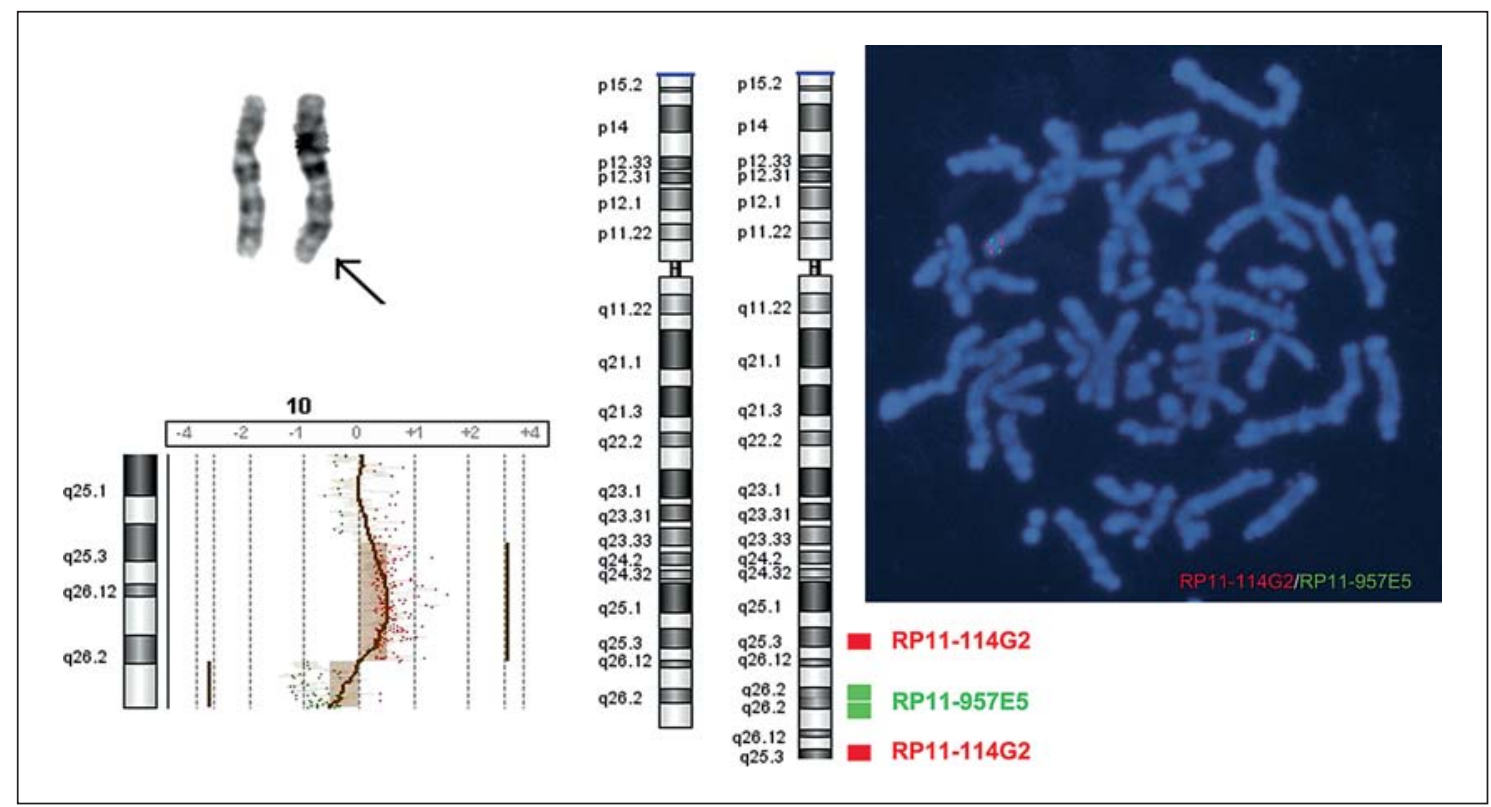

Fig. 2. Left panel: partial karyotype of the patient (GTG-banding). Arrow indicates the derivative chromosome 10 and array-CGH analysis of chromosome 10 shows a duplication in $10 \mathrm{q} 25.3 \mathrm{q} 26.2$ and a deletion in 10q26.2q26.3. Middle panel: partial karyotype idiogram of the patient (GTG-banding). Right panel: FISH analysis shows the double signals with RP11-114G2 probe (red) and RP11-957E5 probe (green) in an inverted position. 


\section{Results}

Metaphase analysis showed that the patient's karyotype was 46,XX,der(10) (fig. 2). The karyotypes of her parents were normal.

Array-CGH analysis demonstrated a $\sim 13.1-\mathrm{Mb}$ duplication encompassing 10q25.3q26.2 (chr10:116,921,304$130,078,056$, hg18) and a $\sim 5-\mathrm{Mb}$ deletion at 10q26.2q26.3 (chr10:130,205,789-135,254,661, hg18) (fig. 2). No singlecopy region was detected between the deleted and duplicated segments.

The analysis of the rearrangement was confirmed by 2 BACs selected in the duplicated regions (RP11-114G2, 10q25.3, bp position: 118,325,684-118,489,992; RP11957E5, 10q26.2, bp position: 127,657,357-127,832,406). Both were found to be duplicated in an inverted position (fig. 2). FISH analysis using the same probes did not show any abnormality in both parents, which indicated a de novo occurrence (data not shown).

Informed written consent for publishing the images of the proband was obtained from her father.

\section{Discussion}

The patient's der(10) was revealed as inv dup del 10q by CGH and FISH analysis. Molecular cytogenetic analysis showed the patient to be trisomic for the $10 \mathrm{q} 25.3 \mathrm{q} 26.2$ segment and monosomic for the terminal 10q26.2q26.3 region. Another 3 patients with inv dup del 10q were also identified and delineated well by microarray technique in combination with other molecular methods, which highlights the importance of these methods in elucidating the complex structural changes [Carter et al., 2010; Kibe et al., 2011; Sarri et al., 2011].

Three mechanisms have been proposed to explain the origin of an inverted duplication with terminal deletion. U-type exchange is the most frequent mechanism for this type of rearrangements in previous patients [Rowe et al., 2009]. The other 2 mechanisms require a single-copy region to exist between the duplicated and deleted regions on the derivative chromosome [Rowe et al., 2009]. No detected disomic region between the duplication and deletion in our patient supports U-type exchange as the most likely mechanism underlying this case.

Most cases of distal 10q duplications were caused by the inheritance of an unbalanced karyotype including additional monosomy of other chromosomes because of familial translocations. Only a few patients belong to the pure distal 10q duplication category. Some previous stud- ies showed that even different partner chromosomes are involved, that the phenotypes of patients can mainly be ascribed to the $10 \mathrm{q}$ duplication and that the concomitant monosomy likely does not influence the clinical features substantially [Briscioli et al., 1993; Bartholdi et al., 2008]. We only included 4 patients with molecularly defined pure distal 10q duplication together with 2 patients with inv dup del 10q and our patient in a phenotype comparision (table 1) [Devriendt et al, 1999; Petek et al., 2001; Migliori et al., 2002; Hou, 2003; Carter et al., 2010; Sarri et al., 2011]. The frequent features of the 4 patients with pure distal 10q duplication involve blepharophimosis (4/4), skeletal anomalies (4/4), hypotonia (3/4), facial dysmorphism (4/4) and developmental delay (4/4).

For the 3 patients with inv dup del $10 \mathrm{q}$, features present in 2 or 3 patients include blepharophimosis (2/3), skeletal anomalies (2/3), hypotonia (3/3), developmental delay $(3 / 3)$, short stature $(2 / 3)$, hypertelorism/epicanthus (3/3), low-set/malformed ears (2/3), strabismus (2/3), and short neck (3/3). In addition to distal 10q duplication, the 3 patients with inv dup del (10q) including ours have concomitant $10 \mathrm{q}$ terminal deletion ranging from $10 \mathrm{q} 26.2 \mathrm{qter}$ (table 1, fig. 3). For patients with 10q26 deletion, the frequent features (expressed in $>50 \%$ of patients) include psychomotor retardation, postnatal growth retardation, cryptorchidism, strabismus, facial dysmorphisms (prominent/broad nasal bridge, low-set/malformed/large/posteriorly rotated ears and hypertelorism), and behavioral anomalies [Waggoner et al., 1999; Scigliano et al., 2004; Courtens et al., 2006]. Our patient and the patient reported by Carter et al. [2010] share many features of distal 10q duplication, such as developmental delay, blepharophimosis, hypotonia, some facial dysmorphisms, absence of major congenital anomalies and skeletal features in the latter, which seems as $10 \mathrm{q}$ duplication accounts for most of the phenotypes. While the patient reported by Sarri et al. [2011] shares many features with pure monosomy 10q26qter, such as facial features, short stature and behavioral traits, other features such as skeletal phenotype and absence of major congenital anomalies are consistent with $10 \mathrm{q}$ duplication patients. The differences might be due to the relatively small duplication fragment of the 10q26.11q26.3 region in the patient reported by Sarri et al. [2011]. Some features such as short stature and hearing loss in the patient reported by Carter et al. [2010] as well as strabismus in our patient also show the phenotypic effect of monosomy 10q26.

Not all the patients present blepharophimosis, which had once been proposed as a characteristic for $10 \mathrm{q}$ duplication, (e.g. a case with 10q26.11q26.2 duplication does 


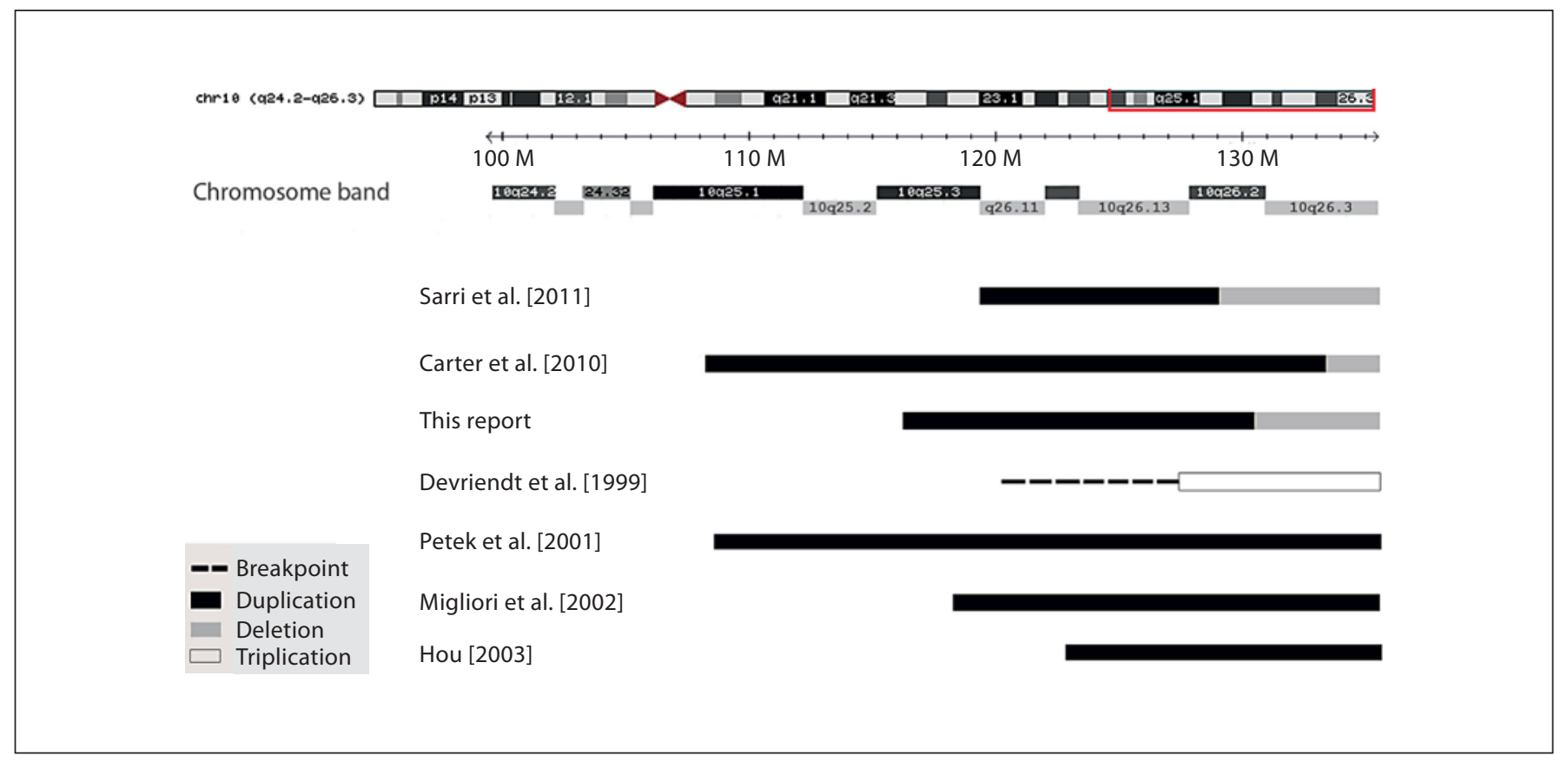

Fig. 3. The affected region of $10 \mathrm{q}$ in our patient. Aberrations in each patient of table 1 are shown by horizontal bars in the lower part.

Table 1. Clinical features of patients with molecularly defined distal 10q duplication

\begin{tabular}{|c|c|c|c|c|c|c|c|}
\hline & Sarri et al. [2011] & Carter et al. [2010] & Ours & Devriendt et al. [1999] & Petek et al. [2001] & Migliori et al. [2002] & Hou [2003] \\
\hline \multicolumn{8}{|l|}{ Segment } \\
\hline Duplication & $10 \mathrm{q} 26.11-\mathrm{q} 26.2$ & $10 \mathrm{q} 25.1-26.3$ & $10 \mathrm{q} 25.3-26.2$ & 10q26-qter & 10q24.33-qter & 10q25.3-qter & 10q26.1-qter \\
\hline \multicolumn{8}{|l|}{ Development } \\
\hline $\begin{array}{l}\text { Mental retardation/ } \\
\text { developmental delay }\end{array}$ & + & + & + & + & + & + & + \\
\hline Blepharophimosis & - & + & + & + & + & + & + \\
\hline Hypertelorism/epicanthus & + & + & + & - & + & + & - \\
\hline Ptosis & - & + & - & - & - & + & - \\
\hline Low-set/malformed ears & - & + & + & - & + & + & - \\
\hline Strabismus & + & - & + & - & - & - & - \\
\hline Short neck & + & + & + & - & - & + & + \\
\hline Hypermobility & - & + & - & + & + & + & + \\
\hline Hip dysplasia & - & + & - & - & + & - & - \\
\hline Hypotonia & + & + & + & + & + & + & - \\
\hline Others & $\begin{array}{l}\text { behavior } \\
\text { anomalies }\end{array}$ & hearing loss & - & - & - & - & $\begin{array}{l}\text { hearing loss; } \\
\text { ventricular } \\
\text { septal defect }\end{array}$ \\
\hline
\end{tabular}


not) [Sarri et al., 2011]. Sarri et al. [2011] and Carter et al. [2010] suggested the dosage-sensitive locus responsible for blepharophimosis resided within band $10 \mathrm{q} 26.2$ or 10q26.3. Our report indicates that the locus contributing to blepharophimosis could be refined to $10 \mathrm{q} 26.2$, a segment between the distal breakpoints of the duplication region of the patient reported by Sarri et al. [2011] and ours. The effect of concomitant terminal deletion may be another explanation. Further factors such as non-penetrant, epigenetic changes or different genetic and environmental factors often play a role in determining the presence or absence of a feature.

Additional frequent features of $10 \mathrm{q}$ duplication are skeletal anomalies, which include camptodactyly/thin finger, sandal gap, scoliosis or hypermobility as listed in table 1. Fibroblast growth factor receptor 2, located at 10q26.13, has been proposed as a probable candidate for skeletal features in the patients [Carter et al., 2010]. Our patient only manifested a moderate laxity of both shoulder joints. Although no obviously abnormal skeletal feature was found in our patient at present, follow-up assessment of skeletal development should be planned with increasing age.

\section{Acknowledgements}

We thank the patient's parents for their participation and support. This work was supported by the project of Shanghai Municipal Natural Science Foundation (grant number 09ZR1425600).

\section{References}

- Bartholdi D, Toelle SP, Steiner B, Boltshauser E, Schinzel A, Riegel M: Blepharophimosis and mental retardation (BMR) phenotypes caused by chromosomal rearrangements: description in a boy with partial trisomy $10 \mathrm{q}$ and monosomy $4 \mathrm{q}$ and review of the literature. Eur J Med Genet 51:113-123 (2008)

- Briscioli V, Floridia G, Rossi E, Selicorni A, Lalatta F, Zuffardi O: Trisomy 10qter confirmed by in situ hybridization. J Med Genet 30:601-603 (1993).

-Carter MT, Dyack S, Richer J: Distal trisomy 10q syndrome: phenotypic features in a child with inverted duplicated 10q25.1-q26.3. Clin Dysmorphol 19:140-145 (2010).

Courtens W, Wuyts W, Rooms L, Pera SB, Wauters J: A subterminal deletion of the long arm of chromosome 10: a clinical report and review. Am J Med Genet A 140:402-409 (2006).

Devriendt K, Matthijs G, Holvoet M, Schoenmakers E, Fryns JP: Triplication of distal chromosome 10q. J Med Genet 36:242-245 (1999).
Hou JW: Chromosomal 10q26 trisomy resulting from paternal $\mathrm{t}(9 ; 10)$ (pter;q26.1). J Formos Med Assoc 102:887-892 (2003).

Kibe T, Mori Y, Okanishi T, Shimojima K, Yokochi K: Two concurrent chromosomal aberrations involving interstitial deletion in 1q24.2q25.2 and inverted duplication and deletion in $10 \mathrm{q} 26$ in a patient with stroke associated with antithrombin deficiency and a patent foramen ovale. Am J Med Genet A 155A:215-220 (2011).

Migliori MV, Ciaschini AM, Discepoli G, Abbasciano V, Barbato M, Pannone E: Distal trisomy of 10q. Report of a new case of duplication 10q25.2-25.3->qter defined by FISH. Ann Genet 45:9-12 (2002).

Petek E, Köstl G, Rauter L, Mutz I, Wagner K, Kroisel PM: Molecular cytogenetics and phenotype characterization of a de novo pure partial trisomy 10(q24.33-qter). Clin Dysmorphol 10:151-153 (2001).

Rowe LR, Lee JY, Rector L, Kaminsky EB, Brothman AR, et al: U-type exchange is the most frequent mechanism for inverted duplication with terminal deletion rearrangements. J Med Genet 46:694-702 (2009).
-Sarri C, Douzgou S, Gyftodimou Y, Tümer Z, Ravn K, et al: Complex distal 10q rearrangement in a girl with mild intellectual disability: follow up of the patient and review of the literature of non-acrocentric satellited chromosomes. Am J Med Genet A 155A:28412854 (2011).

-Scigliano S, Grégoire MJ, Schmitt M, Jonveaux $\mathrm{PH}$, LeHeup B: Terminal deletion of the long arm of chromosome 10. Clin Genet 65:294298 (2004).

Waggoner DJ, Chow CK, Dowton SB, Watson MS: Partial monosomy of distal 10q: Three new cases and a review. Am J Med Genet 86: 1-5 (1999).

-Weleber RG, Verma RS, Kimberling WJ, Fieger HG Jr, Iubs HA: Duplication-deficiency of the short arm of chromosome 8 following artificial insemination. Ann Genet 19:241-247 (1976). 